\title{
Kombucha fermentation using commercial kombucha pellicle and culture broth as starter
}

\author{
Kyu Ri $\mathrm{LEE}^{1 \dagger}$, Kyungae JO ${ }^{1 \dagger}$, Kyung Soo RA², Hyung Joo SUH ${ }^{1 *}$ (D), Ki-Bae HONG ${ }^{3 *}$
}

\begin{abstract}
Kombucha is a fermented beverage containing organic acids by yeast and acetic acid bacteria. In this study, microbial community analysis of kombucha produced in Korea was performed, and changes in components during kombucha fermentation were analyzed using commercial kombucha pellicle and culture broth as starter. The major phylum-level strains of commercial kom-1 and kom-2 showed differences in proteobacteria of $35.60 \%$ and $78.1 \%$, and Firmicutes of $64.06 \%$ and $15.57 \%$, respectively. During fermentation with pellicle (Kom-P) and broth (Kom-F), the level of reducing sugar during fermentation tended to decrease rapidly. The production of acetic acid and D-saccharic acid-1,4-lactone (DSL) in Kom-P and -F tended to increase with increasing fermentation time. In Kom-P, lactic acid and glucuronic acid production increased until 7 days of fermentation and then decreased, whereas in Kom-F, it continued to increase with fermentation time. ABTS radical scavenging activity tended to decrease with increasing fermentation time. However, DPPH radical scavenging activity increased within 7 days of fermentation and then decreased slightly (Kom-P) or remained constant (Kom-F). It has been found that the use of culture broth rather than the use of pellicle as a starter is advantageous to increase the active compound content and DPPH radical scavenging ability.
\end{abstract}

Keywords: kombucha; black tea; radical scavenging activity; DSL; glucuronic acid.

Practical Application: Fermentation of kombucha and its antioxidant activity.

\section{Introduction}

Fermentation is one of the oldest methods to improve food value and preservation via a low-cost energy conservation system. The biochemical changes introduced during fermentation may change the composition of food, thereby imparting new biological activity or increasing digestion and absorption. Recently, fermentation has been used to extract or produce active compounds from plant materials used in the food or beverage industry. Mixed cultures of various microorganisms such as yeast, lactic acid bacteria (LAB), acetic acid bacteria ( $\mathrm{AAB}$ ), and koji fungi (Aspergillus oryzae) have been employed in traditional fermentation industries (Makwana \& Hati, 2019).

Mixed cultures have the advantage of being able to utilize a wide range of enzymes and complex mixtures of carbohydrates, proteins, and fats for fermented food production (Dai et al., 2017). Likewise, the appropriate strain can alter or degrade toxic or noxious compounds that may be present in the fermentation substrate. During food fermentation by mixed microorganisms, yeast produces alcohol, and bacteria produce lactic and organic acids, changing the environment from aerobic to anaerobic; thus, they complement each other and prevent the growth of unwanted microorganisms. Therefore, the inhibitory compounds, anaerobic conditions, and low $\mathrm{pH}$ caused by mixed cultures hinder the production of undesirable mold and bacteria (Hesseltine, 1992).
In Korea, kombucha refers to a black tea fungus and also to a fermented beverage obtained by symbiotic fermentation (bacteria and yeast) of the main raw materials of black tea and sugar (Lee \& Kim, 2000). Kombucha is a healthy carbonated beverage made by fermenting sugar with SCOBY (symbiotic culture of bacteria and yeast) in extracts such as green/black tea (Amarasekara et al., 2020). SCOBY is a culture product of bacteria and yeast used in the manufacturing of various foods and beverages such as kombucha, vinegar, sourdough, and kefir (Gallegos et al., 2016; Laureys et al., 2020). Kombucha is a beverage produced by Saccharomyces and Brettanomyces, which are yeasts that use sugar to produce alcohol, and Acetobacter that use alcohol to produce various organic acids including acetic acid and gluconic acid. It is characterized by the formation of a pellicle, a gel-like membrane of cellulose produced by a symbiotic strain on the surface of the kombucha culture (Jayabalan et al., 2014).

Although despite the various physiological effects of kombucha, its application and utilization in the industry are remarkably low. This is mainly due to insufficient analysis of microbial species, distribution, and metabolites produced during the fermentation of kombucha, which occurs through symbiosis of microorganisms. In this study, in order to industrialize, standardize, and secure the safety of kombucha, the first kombucha fermentation strain and strain distribution were confirmed the changes in several

Received 01 Jan., 2021

Accepted 09 Feb., 2021

${ }^{1}$ Department of Integrated Biomedical and Life Science, Graduate School, Korea University, Seoul, Republic of Korea

${ }^{2}$ Division of Food Beverage and Culinary Arts, Daegu Technical University, Daegu, Republic of Korea

${ }^{3}$ Department of Food Science and Biotechnology, Dongguk University, Goyang, Republic of Korea

*Corresponding author: suh1960@koea.ac.kr, kibae.hong@gmail.com

†These authors contributed equally. 
biochemical markers and antioxidant properties of kombucha fermentation were measured for a period of 3 weeks. Through this study, we intend to provide data that must be secured for the mass production and industrialization of kombucha.

\section{Materials and methods}

Black tea (Lipton, India) was used as a substrate for fermentation, and commercial kombuchas were obtained from Core Bio (Iksan, Korea). A voucher specimen was deposited in Korea University (Seoul, Republic of Kore). ABTS [2,2-azinobis(3-ethylbenzothiazoline-6-sulfonic acid)], DPPH (1,1-diphenyl2-picrylhydrazyl), Folin-Ciocalteu phenol reagent glucuronic acid, and D-saccharic acid-1,4-lactone (DSL) were purchased from Sigma (St. Louis, MO, USA). All chemicals used were HPLC grade or analytical grade.

\subsection{DNA extraction and metagenomic sequencing of $16 \mathrm{~S}$ rRNA}

The liquid samples were centrifuged at $5,000 \times g$ for $10 \mathrm{~min}$, and the supernatant was removed. Kombucha genomic DNA was extracted using the DNeasy PowerFood Microbial kit (Qiagen, Hilden, Germany) according to the manufacturer's protocol. The concentration and purity of metagenomic DNA extracted from kombucha samples were measured using a NanoDrop 1000 spectrophotometer (Thermo Fisher Scientific, Waltham, MA, USA).

For preparation of the MiSeq library amplicons, the $16 \mathrm{~S}$ rRNA V3-V4 variable region of DNA extracted from kombucha samples was amplified. After the first polymerase chain reaction (PCR), the PCR products were purified and a quality-controlled PCR product was used as a template for the second PCR. Nontarget products of purified products from the second PCR were eliminated using Agencourt AMPure XP Beads (Beckman Coulter, Inc.), and the concentration and purity of quantified PCR amplicons were measured using GX caliper HS DNA 1000 (PerkinElmer, Waltham, MA, USA) and the NanoQuant pro200 instrument (Tecan, Mannedorf, Switzerland). Sequencing was carried out at Chunlab, Inc. (Seoul, Republic of Korea) using the MiSeq system (Illumina, San Diego, CA, USA).

Each sequence was analyzed using the EzBioCoud pipeline with EzBioCoud 16S database. Taxonomic ranking and classification were categorized according to the cut-off value criteria [species $(x \geq 97 \%)$, genus ( $97 \%>x \geq 94 \%)$, family $(94 \%>x \geq 90 \%)$, order $(90 \%>x \geq 85 \%)$, class ( $85 \%>x \geq 80 \%)$, and phylum ( $80 \%>x$ $\geq 75 \%$ )] using the EzTaxon-e database (Chun et al., 2007). To compare operational taxonomic units (OTUs) between samples, shared OTUs were obtained with the open-reference method (Yoon et al., 2017).

\subsection{Analytical method}

In order to measure the changes in components during kombucha fermentation, the total sugar and reducing sugar contents were measured using modifications of the phenol-sulfuric acid method (Dubois et al., 1956) and the method reported by Miller (1959), respectively. At this time, glucose was used as a standard when measuring total sugar and reduced sugar. In addition, the polyphenol content was measured by modifying the method proposed by Singleton et al. (1999) using gallic acid as a standard. The organic acid analysis was performed using a Phenomenex Luna C18 column $(250 \times 4.6 \mathrm{~mm}, 5 \mu \mathrm{m})$ using a mixture of potassium dihydrogen phosphate $(\mathrm{pH} 2.4)$ and methanol (97:3) as the mobile phase (flow rate $1.0 \mathrm{~mL} \mathrm{~min}^{-1}$ ) with a UV detector $(220 \mathrm{~nm})$. DSL was determined by HPLC analysis using a YMC C18 column $(250 \times 4.6 \mathrm{~mm}, 5 \mu \mathrm{m})$. Elution was performed using a mobile phase of $3 \%$ methanol in $20 \mathrm{mM}$ $\mathrm{KH}_{2} \mathrm{PO}_{4}\left(\mathrm{pH}\right.$ 2.4) at a flow rate of $1 \mathrm{~mL} \mathrm{~min}{ }^{-1}$, and the elution profile was monitored at $210 \mathrm{~nm}$ with a column temperature of $35^{\circ} \mathrm{C}$ (Chakravorty et al., 2016).

\subsection{Assay of radical scavenging activity}

The DPPH radical scavenging ability was measured according to the method proposed by Cheng et al. (2006) with some modification. After dissolving the sample in ethanol, $800 \mu \mathrm{L}$ was taken and mixed with $200 \mu \mathrm{L}$ of DPPH $(0.15 \mathrm{mM})$ to measure absorbance at $517 \mathrm{~nm}$ after 30 minutes. ABTS radical scavenging ability was measured using the method proposed by Re et al. (1999). Manufactured $7 \mathrm{mM}$ ABTS in which $2.45 \mathrm{mM}$ potassium persulfate was dissolved, it was left in the dark for 12-16 hours, and then diluted with distilled water so that the absorbance at $414 \mathrm{~nm}$ was 1.4-1.5. After reacting $10 \mu \mathrm{L}$ of the sample with $200 \mu \mathrm{L}$ of ABTS (2,2'-azino-bis-3-ethylbenzothiazoline-6sulfonic acid, Sigma-Aldrich) radical solution for 60 minutes, absorbance was measured at $414 \mathrm{~nm}$.

\subsection{Statistical analysis}

Data are presented as mean \pm standard deviation (SD) and the difference between the groups were analyzed using a Student's $t$-test, and differences within the groups were analyzed using one-way analyses of variance (ANOVA) with Tukey's post hoc test. $p$ values $<0.05$ were considered significant.

\section{Results and discussion}

Microbial communities were compared using two types of commercial kombucha (Kom-1 and Kom-2). In kombucha, the major phyla were Proteobacteria $(35.60 \%$ or $78.1 \%)$ and Firmicutes (64.06\% or $15.57 \%)$. Among the microorganisms belonging to the phylum Proteobacteria, Komagataeibacter hansenii and Gluconobacter oxydans were the main species (Figure 1). However, in the phylum Firmicutes, there was a difference between major species in kombuchas. Main species of kombucha Kom-1 were Oenococcus oeni (25.60\%), Lactobacillus (23.1\%), and Sporolactobacillus shoreae (13.04\%). Main species of kombucha Kom-2 were Lactobacillus delbrueckii (6.54\%), Lactobacillus casei (5.45\%), and Ralstonia pickettii (5.81\%) (Figure 1). Five bacterial phyla (Actinobacteria, Bacteroidetes, Deinococcus-Thermus, Firmicutes, and Proteobacteria) were reported from five types of kombucha pellicles. Among them, it was reported that Proteobacteria was the main bacterial phylum (Marsh et al., 2014). The most dominant genus in kombucha was Gluconacetobacter, which represented $85 \%$ or more bacteria in most kombucha. However, the Acetobacter group was 

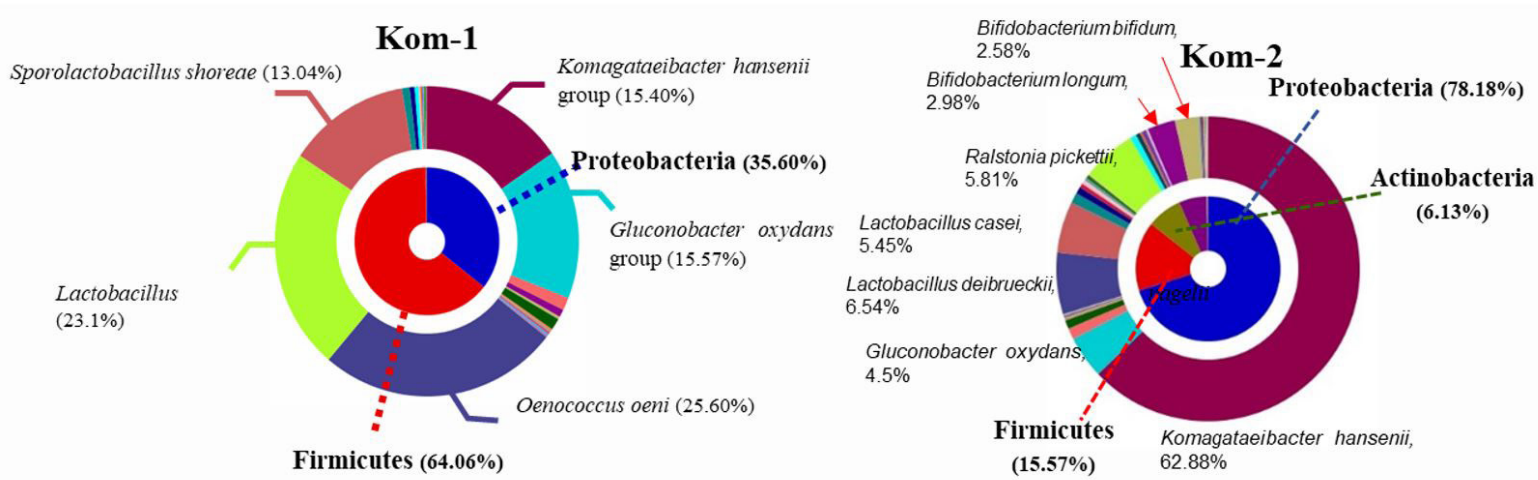

Figure 1. Microbial community in commercial kombucha, Kom-1 and Kom-2. Kom-1: commercial kombucha sample-1; Kom-2: commercial kombucha sample-2.
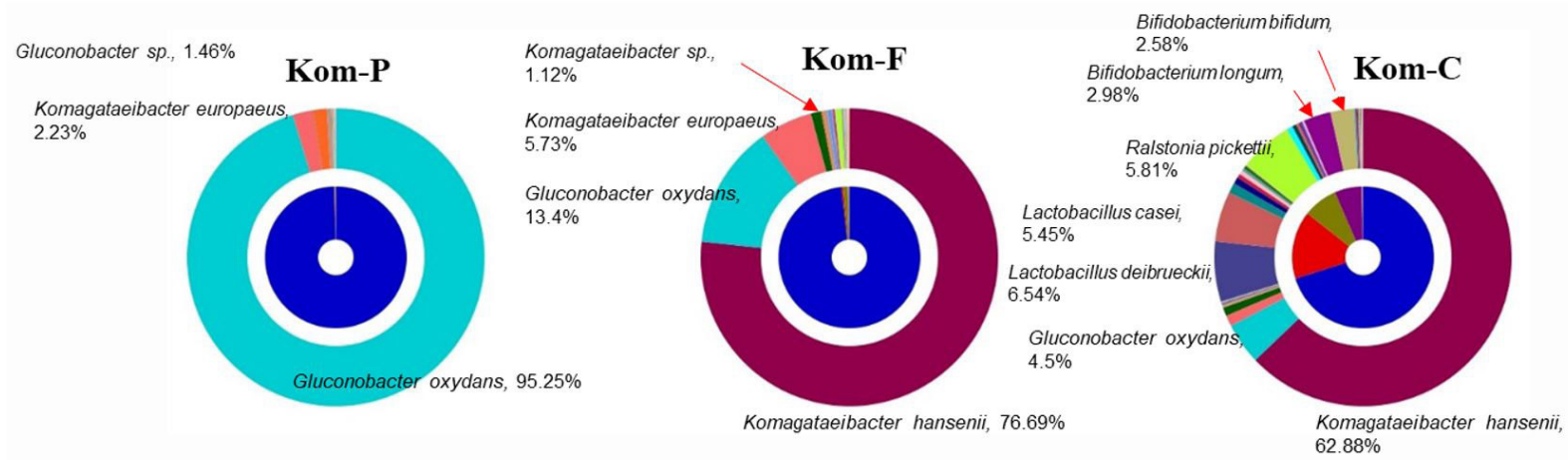

Figure 2. Microbial community in Kom-P, Kom-F, and Kom-C. Kom-P: Kombucha fermented using commercial kombucha (Kom-C) pellicle as a starter; Kom-F: Kombucha fermented using commercial kombucha (Kom-C) fermentation broth as a starter; Kom-C: commercial kombucha.

found to make up less than $2 \%$. There was also a kombucha in which up to $30 \%$ of the Lactobacillus group, known to be non-dominant strains, was detected in kombucha (Marsh et al., 2014). Traditionally, in kombucha, bacteria such as Acetobacter, Gluconacetobacter, and Lactobacillus are the most common species (Trovatti et al., 2011; Yang et al., 2010). Oenococcus oeni, which is rarely observed and is resistant to acid in kombucha (Figure 1), is known to be the most well-known LAB species in wine and apple cider (Breniaux et al., 2018). Although rarely detected in other environments, it has recently been reported in kombucha (Coton et al., 2017). Sporolactobacillus shorea was found in some fermenting materials such as the tree bark in Thailand with spore-forming LAB, but it was first found in kombucha. It shows a different microbiota from the kombucharelated strains reported so far. Microbiota from different pellicles in Europe and the United States observed the highest levels of LAB in Ireland (Marsh et al., 2014). It reports the difference in microbiota of kombucha by region. It is known that these differences in kombucha community are due to differences in $\mathrm{pH}$, temperature, and fermentation substrate.

The kombucha microbial community consist a cellulose biofilm (kombucha pellicle) and a basic liquid or soup (culture broth), so kombucha was prepared using kombucha pellicle and culture broth. Previous reports have shown that kombucha fermentation may differ depending on the inoculum used in the microbial community (Malbaša et al., 2011). Changes in the microbial community were measured after kombucha fermentation by inoculum with different microbiota. (Figure 2). When comparing the phyla, Kom-P (kombucha fermented by pellicle) accounted for $99.77 \%$ of Proteobacteria and $0.18 \%$ of Firmicutes, while Kom- $\mathrm{F}$ (kombucha fermented by fermentation broth) accounted for $99.24 \%$ of Proteobacteria and $0.53 \%$ of Firmicutes. Commercial kombucha (Kom-C) used as a starter accounted for $78.18 \%$ of Proteobacteria, $15.57 \%$ of Firmicutes, and $6.13 \%$ of Actinobacteria. The bacterial diversity of kombucha fermented by inoculating culture broth was higher than that of pellicle. Chakravorty et al. (2016) also reported higher bacterial diversity in the broth than biofilm (pellicle).

Gluconobacter oxydans, which was produced using pellicle as a starter, accounted for $95.25 \%$ of the main fermentation strain in Kom-P. However, kombucha that used fermented broth as a starter contained Komagataeibacter hansenii as the main fermentation strain, which accounted for $76.69 \%$ in Kom-F. Komagataeibacter hansenii, which accounts for $62.88 \%$ of commercial Kombucha (Kom-C), was the main fermentation strain. Komagataeibacter hansenii, identified as the main fermentation bacterium in the second half of fermentation, and also known as Gluconacetobacter hansenii, was identified as an acetic acid-producing bacterium, and is known as a producer of highly crystalline cellulose known as bacterial cellulose. In the heatmap, which plots the abundance and correlation between each OTU at the phylum and species level, the species with the highest number of sequence reads differed 

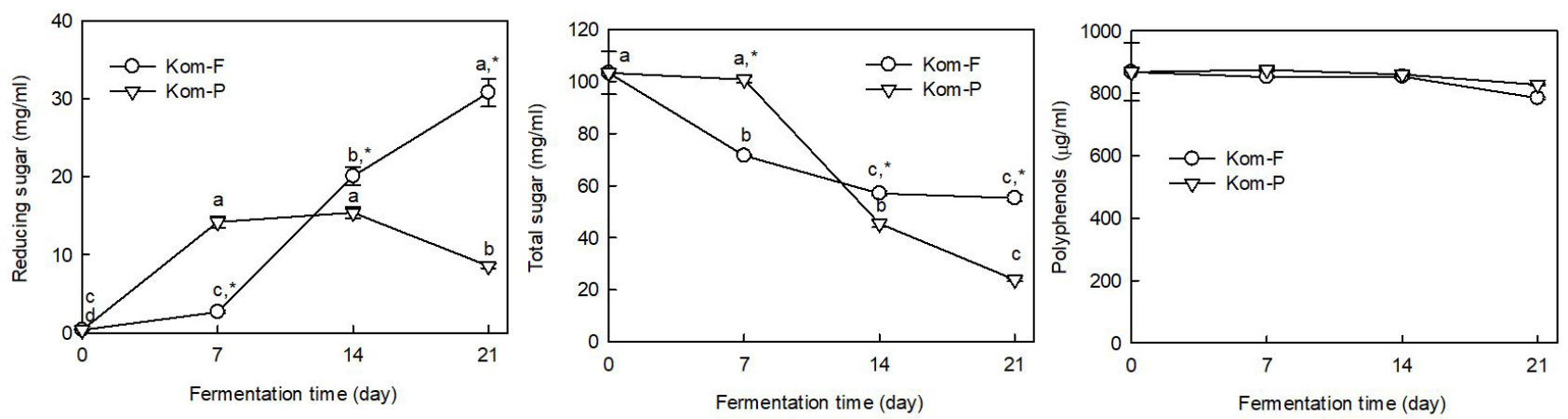

Figure 3. Changes in components during kombucha fermentation with different starters. Kom-P: Kombucha fermented using commercial kombucha (Kom-C) pellicle as a starter; Kom-F: Kombucha fermented using commercial kombucha (Kom-C) fermentation broth as a starter. Different letters indicate significant differences within the group at $p<0.05 .{ }^{*} p<0.05$ indicates a significant difference from other kombucha at the same culture time.

between samples, as shown in Figure 2, kombucha fermented with the culture broth had similarity to commercial kombucha at the phylum and species levels. Similarity was found between Kom-F and KOM-C in the Komagataeibacter and Gluconobacter genera, which were identified as major fermentation strains. In kombucha fermentation, it seems to be more appropriate to use kombucha fermentation starter than pellicle alone.

To confirm the difference between kombucha using a pellicle corresponding to the biofilm as a starter and kombucha using a culture broth as a starter, changes in components according to the culture time were measured. Changes in total sugar and reducing sugar content were measured (Figure 3 ). Changes in total sugar tended to decrease with the increase in fermentation time. Changes in reducing sugar content showed a tendency to increase with fermentation time in Kom-F, while Kom-P slightly increased with fermentation time and then decreased. The total sugar content decreases as fermentation progresses because the fermented strain uses sugar, and because of the tendency for a portion of the reducing sugar to increase as the sugar, which is a non-reducing sugar, is degraded and is converted into a reducing sugar. It is thought that the total sugar content decreases as it is converted to reducing sugar or used by the fermentation strain. Changes in reducing sugar content tended to increase as sugar, a non-reducing sugar, was degraded and converted into reducing sugar. However, the tendency for the levels of reducing sugar to increase and then decrease in Kom-P was the result of reducing sugar, a degradation product of sugar, being used for fermentation in the late fermentation period. Because black tea extract is used as the raw material for kombucha, the initial polyphenol content is high and tends to decrease somewhat by fermentation. In this study, the total polyphenol content was gradually reduced with the fermentation time, which is somewhat different from the reported increase in polyphenol content during fermentation (Jayabalan et al., 2008a, b). The increase in polyphenol content in fermentation occurs because the tea polyphenols containing kombucha seem to be degraded into smaller molecules by enzymes produced by microorganisms involved in the fermentation of kombucha. In particular, in the case of kombucha fermented by Candida sp., such as Candida tropicalis, which is known to be able to degrade complex polyphenols, it is considered that their overall content increases during fermentation because complex polyphenols are enzymatically degraded into small molecules (Ettayebi et al., 2003). However, the kombucha starter used in this study does not seem to have any microorganisms involved in the production of enzymes that can hydrolyze polyphenols into smaller molecules, such as Candida sp.

During kombucha fermentation, acetic acid production tended to increase with increasing fermentation time. The amount of acetic acid produced in Kom-F was higher than that in kombucha fermented with pellicle (Kom-P) as a starter (Figure 4). In addition, the amount of lactic acid increased with the fermentation time in Kom-F, but in the case of Kom-P, lactic acid levels increased until day 7 and then gradually decreased (Figure 4). Kombucha has a sour taste in the early fermentation period, but when the fermentation time increases, the taste changes to a light vinegar-like taste. The main components responsible for this taste are acetic acid and lactic acid, as well as various organic acids (Blanc, 1996). AAB involved in kombucha fermentation use sugar as a carbon source to produce one of the major metabolites, acetic acid. The major AAB involved in kombucha synthesis are Acetobacter, Gluconobacter, and Gluconacetobacter (Komagataeibacter) (Jayabalan et al., 2014). In this experiment, there was a difference in the strains that produce acetic acid due to the difference in starters. The major AAB of Kom-P were Gluconobacter oxydans (95.25\%), and the major AAB of Kom-F were Komagataeibacter hansenii (76.69\%), Gluconobacter oxydans (13.4\%), and Komagataeibacter europaeus $(5.73 \%)$ (Figure 1$)$. The difference in acetic acid and lactic acid production due to the starter difference seems to be due to the difference in the strains involved in fermentation.

The production of glucuronic acid and DSL is shown in Figure 5. When kombucha fermentation used pellicle as a starter, glucuronic acid production at the 7 th day of fermentation showed the highest value at $15.2 \mathrm{mM}$ and then decreased gradually thereafter. However, in Kom-F, glucuronic acid production tended to increase as fermentation time increased. L-Glucuronic acid is another major organic acid found in kombucha metabolites, and production differs depending on the raw material used for preparing kombucha (black tea, green tea, tea manufacture 

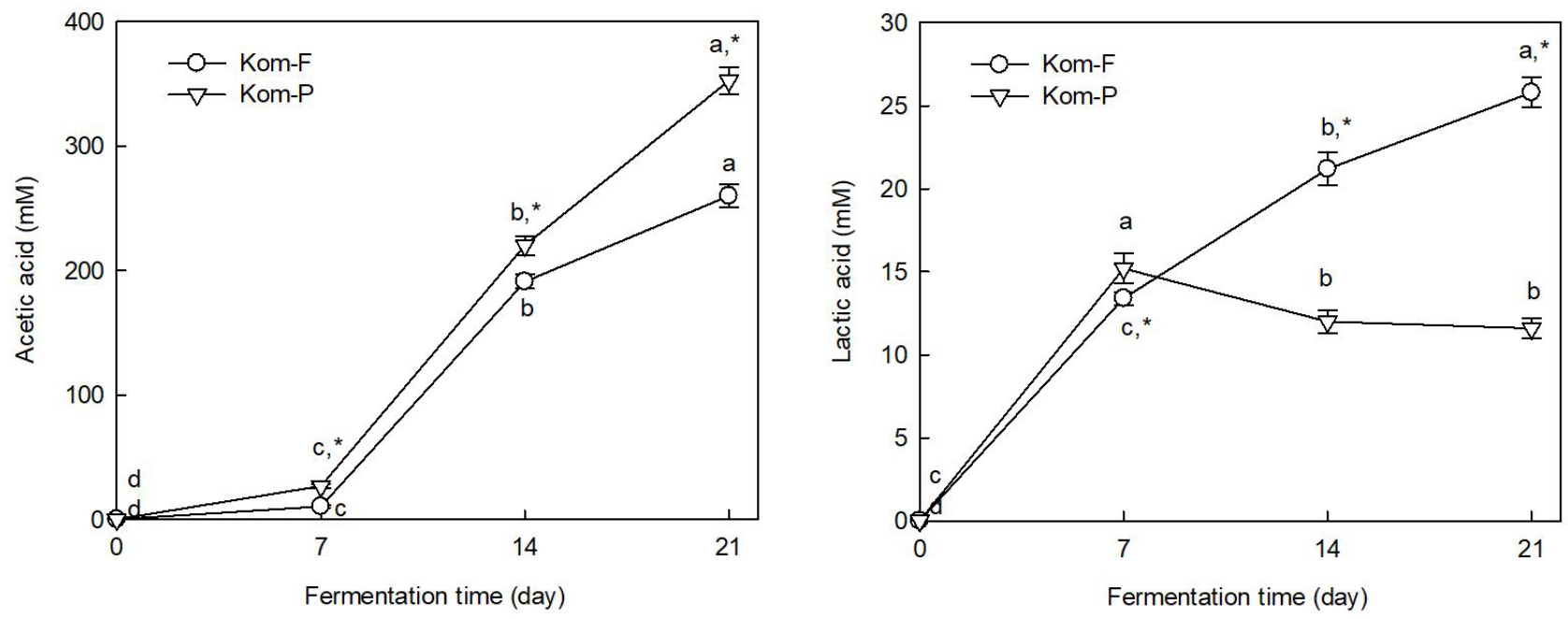

Figure 4. Changes in acetic acid, lactic acid glucuronic acid and DSL contents during kombucha fermentation with different starters. Kom-P: Kombucha fermented using commercial kombucha (Kom-C) pellicle as a starter; Kom-F: Kombucha fermented using commercial kombucha (Kom-C) fermentation broth as a starter. Different letters show significant differences within the group at $p<0.05 .{ }^{\star} p<0.05$ indicates a significant difference from other kombucha at the same culture time.
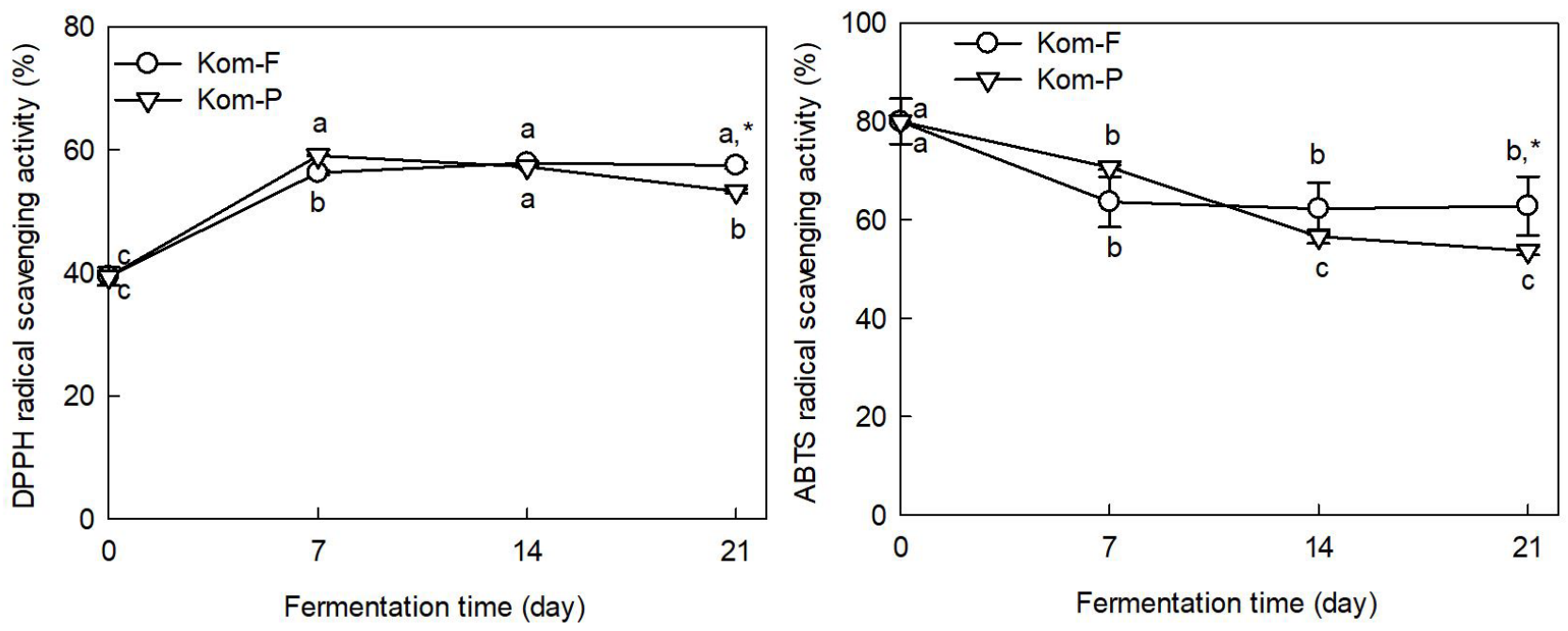

Figure 5. Changes in glucuronic acid and DSL contents during kombucha fermentation with different starters. Kom-P: Kombucha fermented using commercial kombucha (Kom-C) pellicle as a starter; Kom-F: Kombucha fermented using commercial kombucha (Kom-C) fermentation broth as a starter. Different letters show significant differences within the group at $\mathrm{p}<0.05{ }^{*} \mathrm{p}<0.05$ indicates a significant difference from other kombucha at the same culture time.

waste) (Jayabalan et al., 2007). In addition to glucuronic acid, the main active compound in kombucha is DSL. The change in DSL production during fermentation tended to increase with increasing fermentation time (Figure 5). Glucuronic acid and DSL produced during kombucha fermentation contribute to detoxification. In the liver, glucuronic acid combines with toxins to remove them. In addition, DSL inhibits glucuronidase (an enzyme suspected to be the cause of colorectal cancer) in the intestine (Diez \& Cabezas, 1979). DSL serves to inhibit the reuptake of toxins by aiding the breakdown of polysaccharides bound by the enzyme (Kim et al., 1995). Among kombucha fermentation strains, Gluconobacter species can use sugar, but Acetobacter and Gluconacetobacter species use glucose and fructose, the yeast- mediated degradation products of sucrose (Mamlouk \& Gullo, 2013). Therefore, it appears that glucuronic acid increases in fermentation time in Kom-F, which is a predominant bacterium of the genus Gluconacetobacter (Figure 2). In addition, as the main strain of Kom-P, Gluconobacter (Figure 2), uses sucrose directly, it seems that the glucuronic acid content increases and then decreases in the early stage of fermentation. Gluconacetobacter (Yang et al., 2010) and LAB (Wang et al., 2010) are reported to have a strong DSL production capacity. Therefore, it seems that DSL production in Kom-F with many of these strains is high. It seems that the strains involved in kombucha fermentation are important for glucuronic acid and DSL production. 

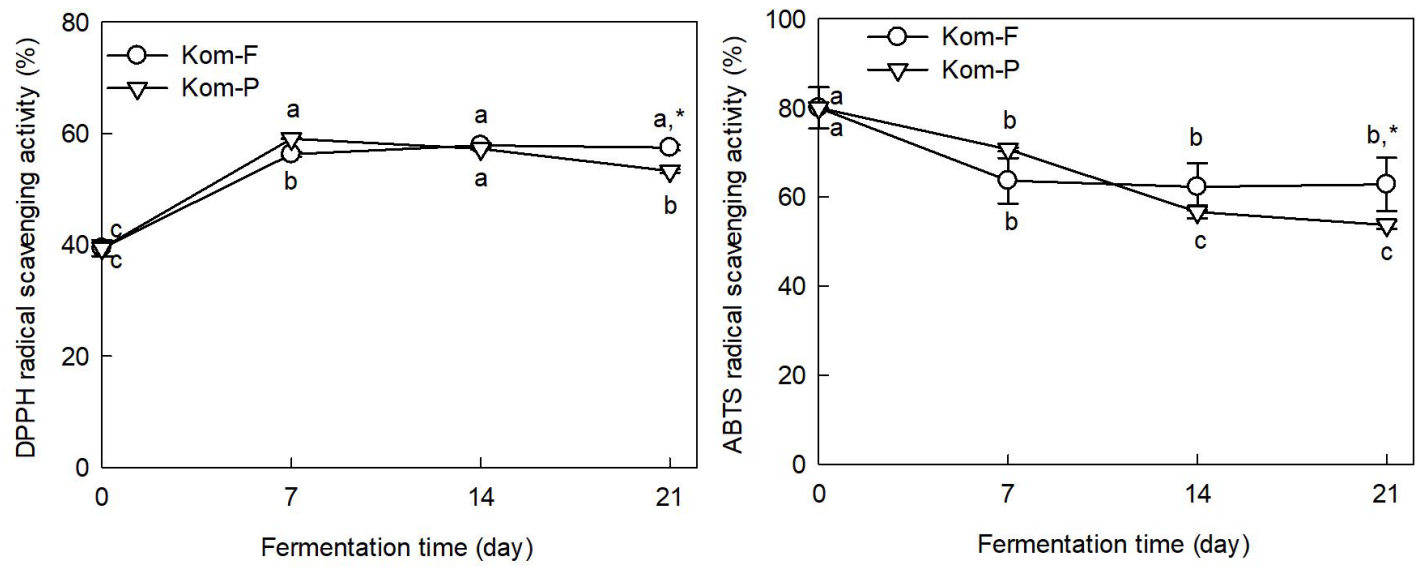

Figure 6. Changes in DPPH and ABTS radical scavenging activity during kombucha fermentation with different starters. Kom-P: Kombucha fermented using commercial kombucha (Kom-C) pellicle as a starter; Kom-F: Kombucha fermented using commercial kombucha (Kom-C) fermentation broth as a starter. Different letters indicate significant differences within the group at $\mathrm{p}<0.05$. ${ }^{*} \mathrm{p}<0.05$ indicates a significant difference from other kombucha at the same culture time.

The radical scavenging activity of kombucha tea represents its potential as a beverage rich in antioxidants. As a result of measuring changes in ABTS or DPPH radical scavenging activity during kombucha fermentation, the ABTS radical scavenging activity tended to decrease with increasing fermentation time (Figure 6). However, DPPH radical scavenging activity increased within 7 days of fermentation and then decreased (Kom-P) or remained constant (Kom-F). The antioxidant activity of kombucha has been attributed to polyphenols, ascorbic acid, and DSL. Thus, kombucha has been observed to have higher antioxidant activity than unfermented tea, which is known to be due to the modification of low molecular weight polyphenol components by enzymes produced by bacteria and yeast during fermentation. However, in this study, the amounts of polyphenols produced during fermentation tended to decrease slightly with fermentation time. This seems to be due to the fact that, as explained above, there are very few Candida species actively produce enzymes involved in the degradation of polyphenols. Jayabalan et al. (2008a, b) reported that the total phenolic compound, the scavenging activity for DPPH radicals, the inhibitory activity for superoxide radicals and hydroxyl radical-mediated linoleic acid were increased during kombucha fermentation, but the reducing power, hydroxyl radical scavenging ability (ascorbic acid-iron EDTA), and anti-lipid peroxidation capacity were reduced. The radical scavenging activity is determined by the fermentation time, the type of tea material, and the characteristics of the metabolites present in the kombucha fermentation strain. The difference in the radical scavenging capacity of kombucha is closely related to the microorganisms involved in fermentation. Microbial diversity affects the formation of metabolites and shows differences in radical scavenging capacity (Liu et al., 1996).

\section{Conclusion}

Currently, kombucha is produced on a small scale through uncontrolled fermentation. To meet the consumer demand, it is important to ensure the reliability of kombucha fermentation. In addition, it is important to establish the process used for kombucha fermentation using isolated strains. Therefore, future research should focus on fermentation using strains isolated from kombucha.

\section{Acknowledgements}

Not applicable.

\section{References}

Amarasekara, A. S., Wang, D., \& Grady, T. L. (2020). A comparison of kombucha SCOBY bacterial cellulose purification methods. SN Applied Sciences, 2(2), 240. http://dx.doi.org/10.1007/s42452-020-1982-2.

Blanc, P. J. (1996). Characterization of the tea fungus metabolites. Biotechnology Letters, 18(2), 139-142. http://dx.doi.org/10.1007/ BF00128667.

Breniaux, M., Dutilh, L., Petrel, M., Gontier, E., Campbell-Sills, H., Deleris-Bou, M., Krieger, S., Teissedre, P. L., Jourdes, M., Reguant, C., \& Lucas, P. (2018). Adaptation of two groups of Oenococcus oeni strains to red and white wines: the role of acidity and phenolic compounds. Journal of Applied Microbiology, 125(4), 1117-1127. http://dx.doi.org/10.1111/jam.13946. PMid:29904988.

Chakravorty, S., Bhattacharya, S., Chatzinotas, A., Chakraborty, W., Bhattacharya, D., \& Gachhui, R. (2016). Kombucha tea fermentation: Microbial and biochemical dynamics. International Journal of Food Microbiology, 220, 63-72. http://dx.doi.org/10.1016/j. ijfoodmicro.2015.12.015. PMid:26796581.

Cheng, Z., Moore, J., \& Yu, L. L. (2006). High-throughput relative DPPH radical scavenging capacity assay. Journal of Agricultural and Food Chemistry, 54(20), 7429-7436. http://dx.doi.org/10.1021/ jf0611668. PMid:17002404.

Chun, J., Lee, J.-H., Jung, Y., Kim, M., Kim, S., Kim, B. K., \& Lim, Y.-W. (2007). EzTaxon: a web-based tool for the identification of prokaryotes based on 16S ribosomal RNA gene sequences. International Journal of Systematic and Evolutionary Microbiology, 57(10), 2259-2261. http://dx.doi.org/10.1099/ijs.0.64915-0. PMid:17911292.

Coton, M., Pawtowski, A., Taminiau, B., Burgaud, G., Deniel, F., Coulloumme-Labarthe, L., Fall, A., Daube, G., \& Coton, E. (2017). Unraveling microbial ecology of industrial-scale Kombucha 
fermentations by metabarcoding and culture-based methods. FEMS Microbiology Ecology, 93(5). http://dx.doi.org/10.1093/femsec/ fix048. PMid:28430940.

Dai, K., Wen, J.-L., Zhang, F., \& Zeng, R. J. (2017). Valuable biochemical production in mixed culture fermentation: fundamentals and process coupling. Applied Microbiology and Biotechnology, 101(17), 65756586. http://dx.doi.org/10.1007/s00253-017-8441-z. PMid:28766035.

Diez, T., \& Cabezas, J. A. (1979). Properties of two molecular forms of $\beta$-glucuronidase from the Mollusc littorina littorea. European Journal of Biochemistry, 93(2), 301-311. http://dx.doi.org/10.1111/j.1432-1033.1979. tb12824.x. PMid:428383.

Dubois, M., Gilles, K. A., Hamilton, J. K., Rebers, P. A., \& Smith, F. (1956). Colorimetric method for determination of sugars and related substances. Analytical Chemistry, 28(3), 350-356. http://dx.doi. org/10.1021/ac60111a017.

Ettayebi, K., Errachidi, F., Jamai, L., Tahri-Jouti, M. A., Sendide, K., \& Ettayebi, M. (2003). Biodegradation of polyphenols with immobilized Candida tropicalis under metabolic induction. FEMS Microbiology Letters, 223(2), 215-219. http://dx.doi.org/10.1016/ S0378-1097(03)00380-X. PMid:12829289.

Gallegos, A. M. A., Herrera Carrera, S., Parra, R., Keshavarz, T., \& Iqbal, H. M. N. (2016). Bacterial cellulose: a sustainable source to develop value-added products. A review. BioResources, 11(2), 5641-5655. http://dx.doi.org/10.15376/biores.11.2.Gallegos.

Hesseltine, C. W. (1992). Mixed-culture fermentations. In National Research Council (Ed.), Applications of biotechnology to traditional fermented foods: report of an ad hoc panel of the Board on Science and Technology for International Development. Washington: National Academies Press.

Jayabalan, R., Malbaša, R. V., Lončar, E. S., Vitas, J. S., \& Sathishkumar, M. (2014). A review on kombucha tea: microbiology, composition, fermentation, beneficial effects, toxicity, and tea fungus. Comprehensive Reviews in Food Science and Food Safety, 13(4), 538-550. http:// dx.doi.org/10.1111/1541-4337.12073. PMid:33412713.

Jayabalan, R., Marimuthu, S., \& Swaminathan, K. (2007). Changes in content of organic acids and tea polyphenols during kombucha tea fermentation. Food Chemistry, 102(1), 392-398. http://dx.doi. org/10.1016/j.foodchem.2006.05.032.

Jayabalan, R., Marimuthu, S., Thangaraj, P., Sathishkumar, M., Binupriya, A. R., Swaminathan, K., \& Yun, S. E. (2008a). Preservation of kombucha tea-effect of temperature on tea components and free radical scavenging properties. Journal of Agricultural and Food Chemistry, 56(19), 9064-9071. http://dx.doi.org/10.1021/jf8020893. PMid:18781766.

Jayabalan, R., Subathradevi, P., Marimuthu, S., Sathishkumar, M., \& Swaminathan, K. (2008b). Changes in free-radical scavenging ability of kombucha tea during fermentation. Food Chemistry, 109(1), 227-234. http://dx.doi.org/10.1016/j.foodchem.2007.12.037. PMid:26054285.

Kim, D. H., Jin, Y. H., Jung, E. A., Han, M. J., \& Kobashi, K. (1995). Purification and characterization of beta-glucuronidase from Escherichia-Coli HGU-3, a human intestinal bacterium. Biological \& Pharmaceutical Bulletin, 18(9), 1184-1188. http://dx.doi.org/10.1248/ bpb.18.1184. PMid:8845801.

Laureys, D., Britton, S. J., \& De Clippeleer, J. (2020). Kombucha tea fermentation: a review. Journal of the American Society of Brewing
Chemists, 78(3), 165-174. http://dx.doi.org/10.1080/03610470.20 20.1734150

Lee, S.-P., \& Kim, C.-S. (2000). Characterization of Kombucha beverages fermented with various teas and tea fungus. Journal of Food Science and Nutrition, 5(3), 165-169.

Liu, C. H., Hsu, W. H., Lee, F. L., \& Liao, C. C. (1996). The isolation and identification of microbes from a fermented tea beverage, Haipao, and their interactions during Haipao fermentation. Food Microbiology, 13(6), 407-415. http://dx.doi.org/10.1006/fmic.1996.0047.

Makwana, M., \& Hati, S. (2019). Fermented beverages and their health benefits. In A. M. Holban \& A. Grumezescu (Eds.), Fermented beverages (pp. 1-29). Duxford: Woodhead Publishing.

Malbaša, R. V., Lončar, E. S., Vitas, J. S., \& Čanadanović-Brunet, J. M. (2011). Influence of starter cultures on the antioxidant activity of kombucha beverage. Food Chemistry, 127(4), 1727-1731. http:// dx.doi.org/10.1016/j.foodchem.2011.02.048.

Mamlouk, D., \& Gullo, M. (2013). Acetic acid bacteria: physiology and carbon sources oxidation. Indian Journal of Microbiology, 53(4), 377384. http://dx.doi.org/10.1007/s12088-013-0414-z. PMid:24426139.

Marsh, A. J., O’Sullivan, O., Hill, C., Ross, R. P., \& Cotter, P. D. (2014). Sequence-based analysis of the bacterial and fungal compositions of multiple kombucha (tea fungus) samples. Food Microbiology, 38 , 171-178. http://dx.doi.org/10.1016/j.fm.2013.09.003. PMid:24290641.

Miller, G. L. (1959). Use of dinitrosalicylic acid reagent for determination of reducing sugar. Analytical Chemistry, 31(3), 426-428. http://dx.doi. org/10.1021/ac60147a030.

Re, R., Pellegrini, N., Proteggente, A., Pannala, A., Yang, M., \& RiceEvans, C. (1999). Antioxidant activity applying an improved ABTS radical cation decolorization assay. Free Radical Biology \& Medicine, 26(9-10), 1231-1237. http://dx.doi.org/10.1016/S0891-5849(98)003153. PMid:10381194.

Singleton, V. L., Orthofer, R., \& Lamuela-Raventos, R. M. (1999). Analysis of total phenols and other oxidation substrates and antioxidants by means of Folin-Ciocalteu reagent. Methods in Enzymology, 299, 152-178. http://dx.doi.org/10.1016/S0076-6879(99)99017-1.

Trovatti, E., Serafim, L. S., Freire, C. S., Silvestre, A. J., \& Pascoal, C. No. (2011). Gluconacetobacter sacchari: an efficient bacterial cellulose cell-factory. Carbohydrate Polymers, 86(3), 1417-1420. http://dx.doi. org/10.1016/j.carbpol.2011.06.046.

Wang, K., Gan, X. H., Tang, X. Y., Wang, S., \& Tan, H. R. (2010). Determination of D-saccharic acid-1,4-lactone from brewed kombucha broth by high-performance capillary electrophoresis. Journal of Chromatography. B, Analytical Technologies in the Biomedical and Life Sciences, 878(3-4), 371-374. http://dx.doi.org/10.1016/j. jchromb.2009.12.003. PMid:20015711.

Yang, Z., Zhou, F., Ji, B., Li, B., Luo, Y., Yang, L., \& Li, T. (2010). Symbiosis between microorganisms from kombucha and kefir: potential significance to the enhancement of kombucha function. Applied Biochemistry and Biotechnology, 160(2), 446-455. http://dx.doi. org/10.1007/s12010-008-8361-6. PMid:18810658.

Yoon, S. H., Ha, S. M., Kwon, S., Lim, J., Kim, Y., Seo, H., \& Chun, J. (2017). Introducing EzBioCloud: a taxonomically united database of $16 \mathrm{~S}$ rRNA gene sequences and whole-genome assemblies. International Journal of Systematic and Evolutionary Microbiology, 67(5), 16131617. http://dx.doi.org/10.1099/ijsem.0.001755. PMid:28005526. 